\title{
Automated vessel centerline extraction and diameter measurement in OCT Angiography
}

\author{
Bernhard K. J. Viertel $^{1}$ \\ Ady Naber ${ }^{1}$ \\ Simon Hoffmann ${ }^{1}$ \\ Daniel Berwanger ${ }^{1}$ \\ Lucy Kessler ${ }^{2}$ \\ Ramin Khoramnia ${ }^{2}$ \\ Werner Nahm ${ }^{1}$ \\ 1 - Institute of Biomedical Engineering, Karlsruhe Institute of Technology, Karlsruhe, Germany, \\ publications@ibt.kit.edu \\ 2 - Department of Ophthalmology, Heidelberg Klinikum, Heidelberg, Germany
}

\section{Introduction}

Optical Coherence Tomography Angiography (OCTA) is a non-invasive imaging technique that enables the visualization of perfused vasculature in vivo. In ophthalmology, it allows the physician to monitor diseases affecting the vascular networks of the retina such as age-related macular degeneration or diabetic retinopathy. Due to the complexity of the vasculature in the retina, it is of interest to automatically extract vascular parameters which describe the condition of the vessels. Suitable parameters could improve the diagnosis and the treatment during the course of therapy.

\section{Methods}

We present an automated algorithm which computes the diameters of the vessels in en face OCTA images. After segmenting the images, the vessel centerline was computed using a thinning algorithm. The centerline was refined by detecting invalid pixels such as spurs and by continuing the centerline until the ends of the vessels. Lastly, the diameter was computed by dilating a discrete circle at the position of the centerline or by measuring the distance between both borders of the vessels. The developed algorithms were applied to in vivo images of human eyes. Certainly, no ground truth was available. Hence, a plausibility check was performed by comparing the measured diameters of two different layers of the retina (Superficial Vascular Complex (SVC) and Deep Vascular Complex (DVC)). Each layer exhibits a different characteristic vasculature.

\section{Results}

The algorithm clearly reflected the differences from both retinal layers. The measured diameters demonstrate that the DVC consists of more capillaries and considerably smaller vessels compared to the SVC.

\section{Conclusion}

The presented method enables automated analysis of the retinal vasculature and forms thereby the basis for monitoring diseases influencing the vasculature of the retina. The validation of the method using an artificial ground truth is still needed. 


\title{
Towards Quantitative ICG Angiography: Fluorescence Monte Carlo Multi Cylinder
}

\author{
Simon Hoffmann ${ }^{1}$ \\ Ady Naber ${ }^{1}$ \\ Werner $\mathrm{Nahm}^{1}$ \\ 1 - Institute of Biomedical Engineering, Karlsruhe Institute of Technology, Karlsruhe, Germany, publications@ibt.kit.edu
}

\section{Introduction}

Intraoperative blood flow measurement is an effective way to assess the quality of bypass surgery. Flow quantification from indocyanine green (ICG) angiography promises to be an easy, contact free method. However, it shows deviations compared to a reference. These are given as factor $\mathrm{k}$, depending on the vessel diameter $\mathrm{d}$. The radiation transport within the vessel while recording the ICG passage might cause this. It is analyzed in silico to disclose its impact on k (d).

\section{Methods}

A Fluorescence Monte Carlo Multi Cylinder (FMCMC) model was developed as a static model, assuming homogeneous concentration of ICG. In contrast to published approaches utilizing a Monte Carlo Multi Layer (MCML) model assuming the deepest penetration location within a photon packet's path to be the fluorescence location, the events were modeled themselves. Flourescence event modeling, Multi Cylinder geometry and a homogeneous illumination as well as combinations of these were implemented in separate aspect models. Resulting k (d) were compared to k (d) from MCML.

\section{Results}

Deviations in $\mathrm{k}(\mathrm{d})$ derived from FMCMC and MCML in each aspect model were present. The Root Square Mean Error ranges from $6,8 \%$ to $36 \%, \mathrm{k}(\mathrm{d})$ also varied comparing the aspect models to each other.

\section{Conclusion}

The model geometry, the modeled fluorescence location and illumination mode show a clear impact on simulated $\mathrm{k}$ (d). Neglecting this by utilizing MCML was proven to be invalid. The developed FMCMC model considers the named aspects, allowing the analysis of radiation transport in ICG angiography.

\section{Outlook}

The FMCMC model assumes a homogeneous concentration of ICG which is not true in clinical cases. Obtaining the heterogeneous distribtion of ICG is possible via fluid flow models. Coupling the fluid flow model and the developed radiation transport model as well as including a detailed camera optic is the task for future works. 


\section{Partial 3D-reconstruction of the colon from monoscopic colonoscopy videos using shape-from-motion and deep learning}

Sina Walluscheck, Fraunhofer IIS and FAU Erlangen, Germany, sina.walluscheck@fau.de

Thomas Wittenberg, Fraunhofer IIS and FAU Erlangen, Germany, thomas.wittenberg@iis.fraunhofer.de

Volker Bruns, Fraunhofer IIS, Erlangen, Germany, volker.bruns@iis.fraunhofer.de

Thomas Eixelberger, IIS, Erlangen, Germany, thomas.eixelberger@iis.fraunhofer.de

Ralf Hackner, Fraunhofer IIS and FAU Erlangen, Erlangen, Germany, ralf.hackner@iis.fraunhofer.de

\section{Introduction}

For the image-based documentation of a colonoscopy procedure, a 3D-reconstuction of the hollow colon structure from the endoscopic video streams is desirable. To obtain this reconstruction, 3D information about the colon has to be extracted from monocular colonoscopy image sequences. This information can be provided by estimating the depth through shape-from-motion approaches, by using the image information from two successive image frames and the exact knowledge of their disparity. Nevertheless, as during a standard colonoscopy the spatial offset between successive frames is continuously changing, in this work deep convolutional neural networks (DCNNs) are applied in order to obtain piecewise depth maps and point clouds of the colon. These pieces can then be fused for a partial 3D reconstruction.

\section{Methods}

Recently, deep convolutional neural network (DCNN) based methods have been suggested and evaluated for various tasks in the field of image analysis in gastroscopy, such as automated adenoma and polyp detection, or differentiation of Barrett's esophagus. For the 3D reconstruction of the colon, or sections thereof, in a first step DCNN-based methods can be applied that approximate depth maps in form of point clouds from temporally sequential colonoscopy image pairs. These 3D segments can then be assembled, registered and fused in a second step [1].

For the step of depth map approximation, three deep neural networks with different architectures, namely 'V-Net', 'U-Net', and 'modified U-Net' (with a ResNet encoder) were constructed and evaluated. For training and validation of these nets, a set of 8,440 successively acquired monoscopic image pairs (captured at $t$ and $t+\Delta t$ ) from a synthetically created (digital) colon model with known disparity and camera geometry were used. Using this data, each of the three networks was trained using 2,000 training epochs, and thus achieved results were quantitatively evaluated against the known ground truth from the colon model. In addition, predicted depth maps of real colonoscopy images were qualitatively evaluated. Based on the visual evaluation, the modified U-Net achieved the best intermediate results. It has a U-Net architecture with a ResNet encoder and was trained for 2,000 epochs on 7,580 training image pairs with 1,485 validation image pairs. For all experiments an Adam optimizer with learning rate $r=1 e-5$, a mean squared loss function and batch size $b=16$ was applied.

\section{Results}

The best results (for the modified U-Net) were achieved for training data consisting of a mixture of synthetic colon images with three different designed surface textures. Integrating real textures, realistic structures and light reflections into the model was beneficial. Additionally, a sufficient amount of camera motion ( $\Delta t=3$ frames) between successive frames of an image pair lead to improved depth estimates, indicating that the shape-from-motion approach is learned by the networks. Furthermore, depth estimates can be computed from an image pair in realtime within $77 \mathrm{~ms}$.

\section{Conclusion}

This work presents a depth estimation approach from monocular colonoscopy images by using deep convolutional neural networks. The DCNNs are trained on synthetic colon data in a supervised manner and can be applied to real colonoscopy videos to generate depth estimates and partial 3D reconstructions in real-time. 


\title{
Multispectral single chip reconstruction using DCNNs with application to open neurosurgery
}

\author{
Stephan Göb, Fraunhofer IIS and FAU Erlangen, Erlangen, Germany, stephan.goeb@gmx.de \\ Theresa Götz, Fraunhofer IIS, Erlangen, Germany, theresa.goetz@iis.fraunhofer.de \\ Thomas Wittenberg, Fraunhofer IIS and FAU Erlangn, Erlangen, Germany, thomas.wittenberg@iis.fraunhofer.de
}

\section{Introduction}

Multispectral imaging (MSI) devices incorporating up to 256 different spectral channels have in the past become available for various healthcare applications. Examples include laparoscopy, gastroscopy, dermatology or perfusion imaging for wound analysis. Currently, the use of such devices is limited due to very high investment costs and slow capture times. In order to compensate these shortcomings, single sensors with spectral masking on the pixel level have been proposed. Hence, adequate spectral reconstruction methods are needed.

\section{Methods}

Within this work, two deep convolutional neural networks (DCNN) architectures with the goal of spectral reconstruction of image data from single sensors are compared with each other. The training of the networks is based on a large collection of different MSI image-stacks, which have been subsampled, simulating 16-channel single sensors with spectral masking. We define a training, validation and test set called HITgoC from different public data sets (HyTexiLa, ICVL, TokyoTech, google and CAVE). Resulting in 351 training (631.128 sub images), 99 validation (163.272 sub images) and 51 test images.

For application in the field of neurosurgery an additional testing set of 36 image stacks from the Nimbus data collection is used, depicing MSI data of the brain during open surgery. The two DCNN architectures were compared to a bilinear interpolation (BI) and an intensity difference (ID) algorithm according to Mihoubi et al.. The DCNNs from Kazumi et al. (ResNet-Shinoda) were trained on HITgoC. The latter consists of a preprocessing step using BI and a refinement part consisting of a ResNet structure. Based on preliminary tests, we replace the BI algorithm by a ID-Interpolation (ResNetID).The similarity measures were Peak Signal-to-Noise Ratio (PSNR), Structural Similarity (SSIM) and Mean-Squared Error (MSE) between the predicted images and the reference images.

\section{Results}

We calculated the similarity measures for the HitgoC and Nimbus data and determined the differences of the mean similarity measure values achieved with the ResNet-ID and baseline algorithms such as BI algorithm and ResNetShinoda. The proposed method achieved significantly better results against BI in SSIM (0.0644 vs. 0.0252), PSNR (15.34 vs. 9.12) and in the $1-\mathrm{MSE}^{*} 100(0.0855$ vs. 0.0273$)$ as well as compared to the ResNet-Shinoda in SSIM (0.0103 vs. 0.0074$)$, PSNR (3.87 vs. 3.66) and 1-MSE*100 (0.0075 vs. 0.0047) for HITgoC/Nimbus.

\section{Conclusion}

In this study, significantly better results for spectral reconstruction in MSI images of open neurosurgery was achieved using a combination of ID-Interpolation and a ResNet structure compared to standard methods. 


\section{PTT-based Contact-less Blood Pressure Measurement using an RGB- Camera}

Carolin Wuerich, Fraunhofer IMS, Duisburg, Germany, carolin.wuerich@ims.fraunhofer.de; Robin Rademacher, Fraunhofer IMS, Duisburg, Germany, robin.rademacher@rwth-aachen.de; Christian Wiede, Fraunhofer IMS, Duisburg, Germany, christian.wiede@ims.fraunhofer.de; Anton Grabmaier, Department of Electronic Components and Circuits, University of Duisburg-Essen, Duisburg, Germany, anton.grabmaier@ims.fraunhofer.de;

\section{Introduction}

Although blood pressure is one of the most fundamental vital parameters for evaluating the health status of a person, state-of-the-art measurement methods have noticeable limitations. Cuff-based systems are uncomfortable for patients, provide only intermitted measurements and have a comparatively high error margin. Continuous cuff-less measurement devices are mostly invasive and pose a high risk for infections or thrombosis. To overcome these limitations, we developed and tested a surrogate-based non-invasive blood pressure measurement method using an RGB-camera.

\section{Methods}

Our proposed method employs the relation between the pulse transit time (PTT) and blood pressure. Therefore, we start with a chromatic adaption to provide a constant illumination throughout the video. Then, we detect and track two skin regions, one in the face and the other one on the palm, to extract two remote photoplethysmography (rPPG) signals at different body locations with different distances from the heart. After denoising the signals, the zero crossings are detected and finally the PTT is calculated as the temporal delay of the zero crossings between these two regions. To establish the correlation between the PTT values and the blood pressure, different regression models are trained and evaluated.

\section{Results}

Tests were performed with eight subjects between the age of 18 and 62, where each subject was recorded three times for 30 seconds each. Before and after the recording, reference measurements were taken using a cuff-based sphygmomanometer. Since the phyiological parameters of the cardiac system are different for each person, an individual calibration is required to obtain the systolic and diastolic blood pressure from the PTT values.

\section{Conclusion}

The calibration results are limited by the small number of samples, however, the results show a strong correlation between the PTT values and the blood pressure. For the future, we are going to record videos with beat-to-beat reference data to enable a more effective training. 


\section{Learning about reflective PPG for $\mathrm{SpO}_{2}$ determination using Machine Learning}

Idoia Badiola Aguirregomezcorta, Lehrstuhl für Medizinische Informationstechnik (MedIT), RWTH Aachen University, Aachen, Germany, badiola@ hia.rwth-aachen.de

Vladimir Blazek, Steffen Leonhardt, Lehrstuhl für Medizinische Informationstechnik (MedIT), RWTH Aachen University, Aachen, Germany

Christoph Hoog Antink, Fachgebiet Medizintechnik KIS*MED, Technische Universität Darmstadt, Darmstadt, Germany, hoogantink@kismed.tu-darmstadt.de, formerly Lehrstuhl für Medizinische Informationstechnik (MedIT), RWTH Aachen University, Aachen, Germany

\section{Introduction}

Reflective Photoplethysmography (PPG) sensors are less obtrusive and less exposed to perfusion changes than transmissive sensors, but they present patient-dependent variations in the so-called "Ratio of Modulation" $(R)$. Thus, the conventionally employed calibration curves for determining peripheral oxygen saturation $\left(\mathrm{SpO}_{2}\right)$ may report inaccurate values. In this paper, we study the possibility of overcoming these limitations through Machine Learning (ML). For that, we show the results of applying several algorithms and feature combinations to PPG data from a human hypoxia study.

\section{Methods}

The hypoxia study was performed on ten healthy subjects. Their target oxygen saturation was reduced in five steps from 98-100\% to 70-77\% through an oral mask. Blood Gas Analysis (BGA) was performed five times for each saturation level to measure the arterial oxygen saturation. PPG data were acquired from a reflective pulse oximeter placed in the subjects' ear canals.

PPG signals recorded at $760 \mathrm{~nm}$ and $900 \mathrm{~nm}$ were pre-processed, and several features in the frequency and temporal domain were calculated. For the ML algorithms' input, we explored different combinations of the features. We trained and validated the algorithms with the data from seven patients, and we tested them on three. Finally, we performed leave-one-out cross-validation to ensure the universality of the methods.

\section{Results}

The results show a good agreement of the predictions with the BGA values for Linear Regression, k-Nearest Neighbors, Stochastic Gradient Descent, and Neural Network for all input feature combinations with an average RMSE in the range of 3\%. However, the performance of the Linear Regression was not beaten by the Neural Network, even for overfitting with 2000 hidden layers. The combination of $R$ calculated with a Fast-Fourier Transform and $A C_{\text {RMSred }} / A C_{\text {RMSir }}$ significantly improved the results, reducing the RMSE by $25 \%$.

\section{Conclusion}

This work demonstrates that a straight-forward Linear Regression is capable of determining $\mathrm{SpO}_{2}$ with reflective PPG independently of the subject if the ratio $A C_{\text {RMsred }} / A C_{\text {RMsir }}$ is considered simultaneously with the Ratio of Modulation $R$. 